CASE REPORT

\title{
Carcinoma metastasis to unexpected synchronous lymphoproliferative disorder: report of three cases and review of literature
}

\author{
U Pandey, M Naraynan, U Karnik, B Sinha
}

J Clin Pathol 2003;56:970-971

Metastasis of one cancer to another cancer is rare. This report describes three cases in which low grade non-Hodgkin lymphomas were incidentally detected during the examination of lymph nodes removed as part of treatment of a previously diagnosed malignancy.

$\mathrm{T}$ he occurrence of multiple, primary tumours in the same individual is well recognised. ${ }^{12}$ However, the simultaneous occurrence of two malignancies involving one organ/site is rare. ${ }^{3}$ The second malignancy may present as an unexpected finding during the investigation of the previous malignancy. We report three cases where lymph node metastasis of a previously diagnosed carcinoma was seen together with an unexpected lymphoproliferative disorder.

\section{CASE REPORTS}

\section{Case 1}

A wide local excision and axillary node clearance was carried out on a 75 year old woman for established carcinoma of the left breast. Histology revealed the presence of metastatic carcinoma in three nodes. In addition, the nodes revealed a neoplastic proliferation of small lymphocytes expressing a B cell phenotype. Immunophenotyping results of peripheral blood mononuclear cells were typical of B cell chronic lymphocytic leukaemia (CLL). She received local radiotherapy for the breast cancer but no treatment was given for the CLL. At six months follow up, there was no evidence of progression of the CLL.

\section{Case 2}

Left neck dissection was performed on an 82 year old man as part of the treatment for previously diagnosed Merkel cell carcinoma of the nasal skin. Seven nodes revealed metastatic deposits confirmed with positive immunostaining for Cam 5.2 and chromogranin (fig 1). The background nodal architecture was effaced by a diffuse infiltrate of small lymphoid cells expressing a B cell phenotype. Surface marker analysis results were typical of a B cell CLL. No treatment was given for the CLL. Local recurrence of the Merkel cell carcinoma was seen at 12 months follow up but there was no evidence of progression of the CLL.

\section{Case 3}

An 83 year old man underwent right side neck dissection for a previously diagnosed squamous cell carcinoma of the right pinna. Metastatic carcinoma was seen in 18 nodes (fig 2). In addition, the nodes revealed an unexpected B cell small lymphocytic lymphoma (SLL). Bone marrow trephine revealed a small interstitial infiltrate of the non-Hodgkin lymphoma (NHL). There was no systemic disease and the patient did not receive treatment for the NHL.
The patient died of metastatic squamous cell carcinoma eight months after the first diagnosis.

\section{DISCUSSION}

Synchronous tumours (diagnosed within a six month period) are rare. ${ }^{4}$

In our three patients, the presenting/symptomatic malignancy was a breast carcinoma, Merkel cell carcinoma, and cutaneous squamous carcinoma, respectively. An unexpected low grade lymphoproliferative disorder in the form of CLL/ SLL was detected during histopathological examination of the resected lymph nodes. Because none of our patients had received previous chemotherapy or radiotherapy, it was likely that the CLL/SLL was a true synchronous neoplasm, unrelated to treatment of the carcinoma.

The association of CLL/SLL with another primary malignancy has been noted by several authors. ${ }^{4}{ }^{5}$ Impairment of the immune system caused by CLL could be a predisposing factor. Various factors have been suggested to contribute to the occurrence of multiple synchronous neoplasms (not related to treatment). These include genetic susceptibility, advanced age of the patients, depressed cellular immunity (immunosuppression produced by the first tumour), and exposure to a common inducing agent. ${ }^{46}$

Metastasis of one malignancy to tissue involved by another malignancy is uncommon. Cancers of the breast and skin are among those reported to metastasise to nodes involved by lymphoma. ${ }^{6-8}$ Cutaneous tumours associated with lymphoproliferative disorders have been documented in the literature. ${ }^{4}$ Recently, Merkel cell carcinoma has been described in patients with CLL. ${ }^{10}{ }^{11}$ However, to the best of our knowledge, a case such as ours, in which Merkel cell carcinoma metastasised to a lymphomatous lymph node, has not been reported in the literature. This is especially important because, as a result of apparent morphological similarity, the presence of a coexistent lymphoma may be overlooked.

\section{"Our cases highlight that lymph node enlargement in patients with a known primary malignancy does not always indicate metastasis"}

Growth and survival of a tumour in any new organ is dependant on the microenvironmental influences at that site, including the interaction of growth factors, effects of extracellular matrix, adhesion molecules, etc. Metastasis of carcinoma to lymphomatous lymph nodes is rare. Obliteration of lymphatic sinuses by the neoplastic lymphoid population has been cited as a possible factor. ${ }^{6}$ Alternatively,

Abbreviations: CLL, chronic lymphocytic leukaemia; NHL, non-Hodgkin lymphoma; SCC, small lymphocytic lymphoma 

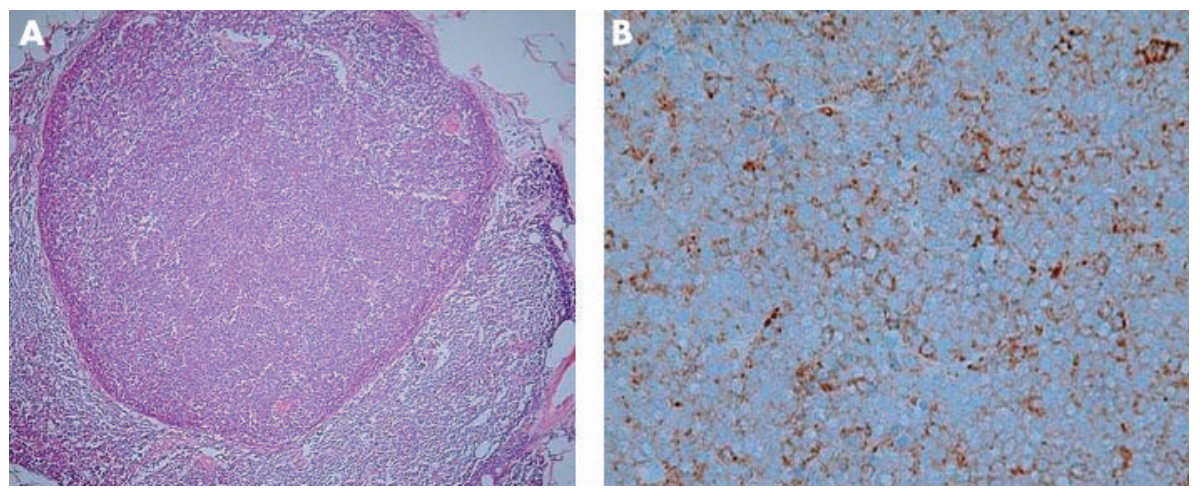

Figure 1 (A) Haemotoxylin and eosin stained section showing metastasis of Merkel cell carcinoma to a lymph node affected by $B$ cell chronic lymphocytic leukaemia. (B) Merkel cell tumour cells staining positively for chromogranin.
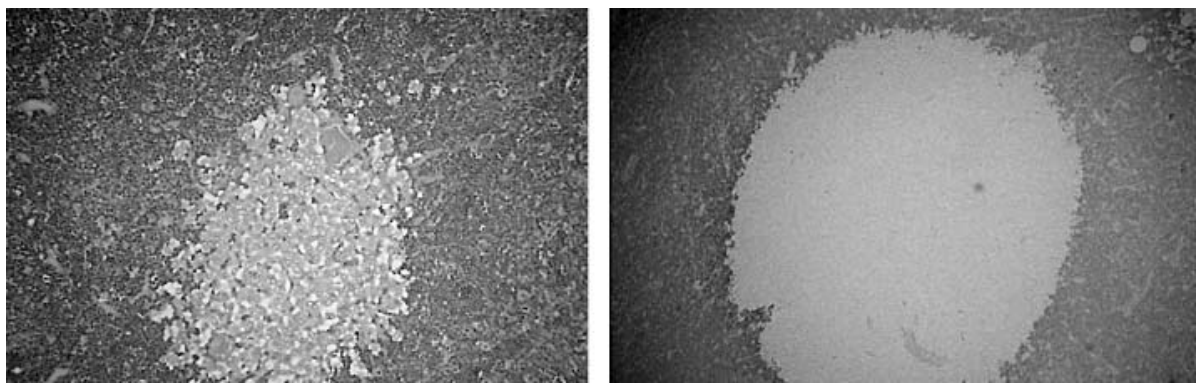

Figure 2 Metastatic deposit of carcinoma cells in a lymph node involved by $B$ cell chronic lymphocytic leukaemia (left). CD79a (right) highlights the lymphoma cells.

lymphoma cells may produce substances locally antagonistic to the second malignancy. However, our cases illustrate that the mechanism is complex because occasionally carcinoma does metastasise to lymphomatous nodes. It is likely that, in these cases, the lymphatic channels are at least partially patent and the carcinoma cells overcome resistance offered by the pre-existing lymphoma cells through a complex interaction between chemokines.

Our cases highlight that lymph node enlargement in patients with a known primary malignancy does not always indicate metastasis. Apart from documenting the coexistence of a Merkel cell carcinoma and lymphoma in the same lymph node, the other principal purpose of our article is to alert clinicians and pathologists to the possibility of the coexistence of dual malignancies to prevent inaccurate staging of the first malignancy and delay in diagnosis of the second malignancy. This may have therapeutic implications depending on the nature of the second malignancy. The

\section{Take home messages}

- We believe that this is the first report of a Merkel cell carcinoma metastasising to a lymphomatous lymph node

- Clinicians and pathologists should be aware that two different malignancies can coexist in the same organ

- Such an awareness will prevent inaccurate staging of the first malignancy and delay in diagnosis of the second malignancy

- There may be therapeutic implications depending on the nature of the second malignancy

- Chronic lymphocytic leukaemia may run a benign course, so that adjuvant treatment for the other malignancy need not be withheld natural history of CLL is variable, and it may run a benign course. Hence, especially in old patients, as in our cases, adjuvant treatment for the other malignancy need not be withheld.

\section{Authors' affiliations}

U Pandey, M Naraynan, B Sinha, Department of Pathology, George Eliot Hospital NHS Trust, College Street, Nuneaton CV10 7DJ, UK U Karnik, Royal Wolverhampton Hospitals, Wolverhampton WV10 OQP, UK

Correspondence to: Dr U C Pandey, George Eliot Hospital NHS Trust, College Street, Nuneaton CV10 7DJ, UK; mila@upandey.fsnet.co.uk

Accepted for publication 30 May 2003

\section{REFERENCES}

1 Warren S, Gates O. Multiple primary malignant tumours. A survey of the literature and a statistical study. Am J Cancer 1932;16:1358-414.

2 Watson TA. Incidence of multiple cancer. Cancer 1953;6:365-71.

3 Rabson SM, Stier PL, Baumgartner JC, et al. Metastasis of cancer to cancer. Am J Clin Pathol 1954:24:572-9.

4 Lee TK, Myers RT, Scharyj M, et al. Multiple primary malignant tumours (MPMT): study of 68 autopsy cases (1963-1980). J Am Geriatr Soc 1982;30:744-53.

5 Moertel CG, Hagedorn AB. Leukemia or lymphoma and coexistent primary malignant lesions: a review of the literature and a study of 120 cases. Blood $1957 \cdot 12 \cdot 788-803$

6 Ambrosiani L, Bellone S, Dechlich P, et al. Breast carcinoma metastatic to a lymphoma: report of a case with review of literature. Histopathology 1999:34:87-9.

7 Perez-Reyes N, Farhi DC. Squamous cell carcinoma of head and neck in patients with well differentiated lymphocytic lymphoma. Cancer 1987;59:540-4.

8 Caraway NP, Wojcik EM, Saboorian HM, et al. Concomitant lymphoma and metastatic carcinoma in a lymph node: diagnosis by fine needle aspiration biopsy in two cases. Diagn Cytopathol 1997; 17:287-91.

9 Levi F, Randimbison L, Te V-C, et al. Non-Hodgkin's lymphomas, chronic lymphocytic leukemias and skin cancers. Br J Cancer 1996;74:1847-50.

10 Ziprin P, Smith S, Salerno G, et al. Two cases of merkel cell tumour arising in patients with chronic lymphocytic leukaemia. Br J Dermatol 2000:142.525-8.

11 Cottoni F, Montesu MA, Lissia A, et al. Merkel cell carcinoma, Kaposi's sarcoma, basal cell carcinoma and keratoacanthoma: multiple association in a patient with chronic lymphatic leukaemia. Br J Dermatol 2002; 147:1029-31. 